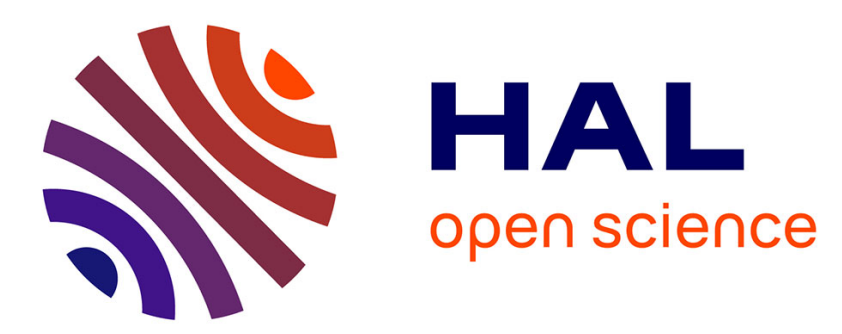

\title{
rEXPAR: An Isothermal Amplification Scheme That Is Robust to Autocatalytic Parasites
}

\author{
Georg Urtel, Marc van Der Hofstadt, Jean-Christophe Galas, André
}

Estévez-Torres

\section{- To cite this version:}

Georg Urtel, Marc van Der Hofstadt, Jean-Christophe Galas, André Estévez-Torres. rEXPAR: An Isothermal Amplification Scheme That Is Robust to Autocatalytic Parasites. Biochemistry, 2019, 58 (23), pp.2675-2681. 10.1021/acs.biochem.9b00063 . hal-02180838

\section{HAL Id: hal-02180838 \\ https: / hal.sorbonne-universite.fr/hal-02180838}

Submitted on 11 Jul 2019

HAL is a multi-disciplinary open access archive for the deposit and dissemination of scientific research documents, whether they are published or not. The documents may come from teaching and research institutions in France or abroad, or from public or private research centers.
L'archive ouverte pluridisciplinaire HAL, est destinée au dépôt et à la diffusion de documents scientifiques de niveau recherche, publiés ou non, émanant des établissements d'enseignement et de recherche français ou étrangers, des laboratoires publics ou privés. 


\title{
rEXPAR: an isothermal amplification scheme that is robust to autocatalytic parasites
}

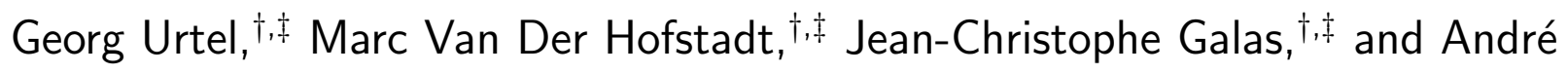 \\ Estevez-Torres*,†, $\ddagger$ \\ †Sorbonne Université, Laboratoire Jean Perrin, F-75005, Paris, France \\ $\ddagger U M R$ 8237, CNRS, F-75005, Paris, France \\ E-mail: andre.estevez-torres@upmc.fr
}

\begin{abstract}
In the absence of DNA, a solution containing the four deoxynucleotidetriphosphates (dNTPs), a DNA polymerase and a nicking enzyme generates a self-replicating mixture of DNA species called parasite. Parasites are problematic in template-based isothermal amplification schemes such as EXPAR, as well as in related molecular programming approaches, such as the PEN DNA toolbox. Here we show that using a nicking enzyme with only three letters $(\mathrm{C}, \mathrm{G}, \mathrm{T})$ in the top strand of its recognition site, such as Nb.BssSI, allows to change the sequence design of EXPAR templates in a way that prevents the formation of parasites when dATP is removed from the solution. This method allows to make the EXPAR reaction robust to parasite contamination, a common feature in the laboratory, while keeping it compatible with PEN programs, which we demonstrate by engineering a parasite-proof bistable reaction network.
\end{abstract}

\section{Keywords}

in vitro synthetic biology, DNA nanotechnology, isothermal amplification 


\section{Introduction}

Isothermal nucleic acid amplification methods ${ }^{1}$ are interesting alternatives to the polymerase chain reaction (PCR) because they do not need thermocycling equipment and are thus suited for the detection of nucleic acids in resource-limited environments. ${ }^{2}$ Different molecular implementations exist, such as nucleic acid sequence-based amplification (NASBA), strand displacement amplification (SDA), rolling circle amplification (RCA), loop-mediated isothermal amplification (LAMP) and exponential amplification reaction (EXPAR). ${ }^{3}$

Among the cited methods, EXPAR has two important advantages. Firstly, it has a short detection time in the order of minutes. ${ }^{4}$ Secondly, it produces single stranded DNA (ssDNA) as an output which can be used, either to set up simple colorimetric detection methods based on the aggregation of DNA-decorated nanoparticles, ${ }^{5}$ or to couple EXPAR to molecular programs capable of displaying complex spatiotemporal dynamics. ${ }^{6-9}$ EXPAR exponentially amplifies the concentration of a trigger ssDNA $\mathbf{A}$ in the presence of a ssDNA template $\mathbf{T}$, a DNA polymerase and a nicking endonuclease, called nickase in the following (Figure 1a).

However, EXPAR has two important drawbacks induced by non-specific reactions. The first one, usually known as early phase background amplification, ${ }^{4}$ or self-start, limits the detection of very low quantities of DNA. Several solutions have recently been proposed for this problem. ${ }^{10-12}$ The second problem, known as late-phase background amplification, ${ }^{4}$ or untemplated amplification, arises in systems where nucleic acids are exponentially amplified with the help of enzymes. Mutations lead to new sequences and, after some time, a parasitic sequence - or set of sequences - emerges, which is able to replicate more efficiently than the initial target sequence. One famous example is Sol Spiegelman's monster, that arouse during the in vitro replication of $\mathrm{Q} \beta$-RNA with $\mathrm{Q} \beta$-replicase and nucleotides. ${ }^{13}$ The EXPAR reaction produces a different kind of parasitic species containing repetitive and palindromic sequences where, typically, AT tracts are flanked by nickase recognition sites. ${ }^{4}$ Importantly, parasites appear by de novo, or untemplated, synthesis of DNA. Autocatalytic parasites have 
been observed in the presence of enzymes other than nickases, such as restriction enzymes, ${ }^{14}$ helicases ${ }^{15}$ and possibly T7 RNA polymerase, ${ }^{16}$ and also in PCR reactions either as a sideeffect $^{17}$ or by design. ${ }^{18}$

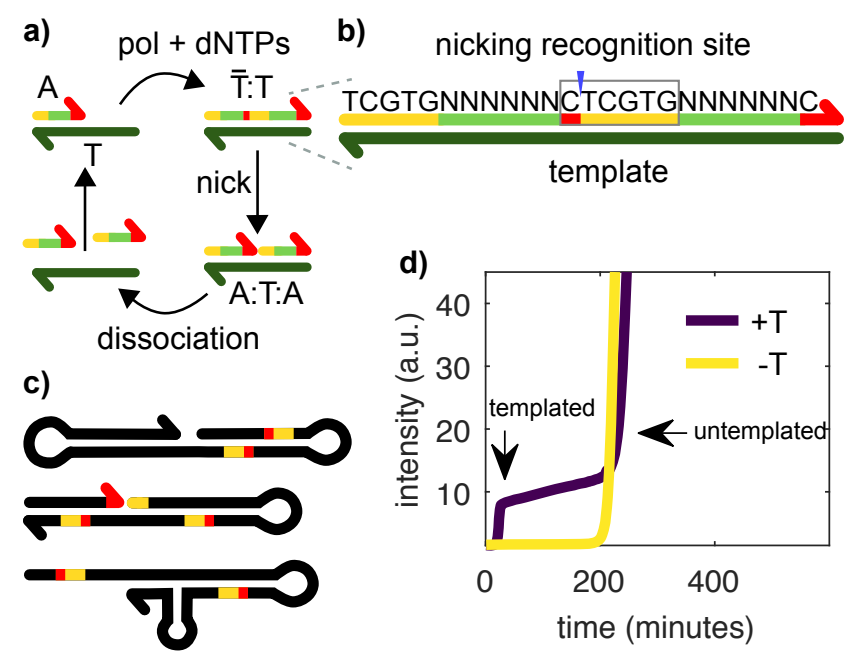

Figure 1: Templated and untemplated replication in the EXPAR reaction. a) During templated replication, the trigger $\mathbf{A}$ is elongated on the template $\mathbf{T}$ by a polymerase (pol), consuming dNTPs. The double stranded complex $\overline{\mathbf{T}}: \mathbf{T}$ is nicked by a nickase (nick) and two As are created, which can dissociate and replicate on other Ts. b) Each trigger contains a split-up recognition site for the nickase (red and yellow), which is completed after elongation. The sequence of the six $\mathrm{N}$ nucleotides can be chosen freely. c) In EXPAR experiments an untemplated replicator, termed the parasite, emerges after some time. The parasite is not a single sequence, but a pool of sequences. They are rich in secondary structures and bear recognition sites for the nickase. Unlike shown here, they can reach several kbp in length. d) EvaGreen fluorescence $v s$. time for an EXPAR reaction in the presence $(+\mathbf{T}$, purple) and in the absence $\left(-\mathbf{T}\right.$, yellow) of template strand $\mathbf{T}_{1}$. EvaGreen is a DNA intercalator allowing to track the concentration of dsDNA

Because parasite replication is as efficient as templated replication (SI Figure S5), parasites easily contaminate the whole laboratory and are difficult to eradicate, thus posing a problem to make EXPAR a robust analytical technique. Furthermore, when EXPAR is used to build more complex molecular programs, such as oscillators or bistable networks, the emergence of parasites limits the lifetime of these systems to typically one day, restricting 
the use of these powerful molecular programs ${ }^{19}$ for building non-equilibrium materials. ${ }^{9}$ In this work, we show that choosing a nickase with only three letters $(C, G, T)$ in the top strand of its recognition site, such as Nb.BssSI, allows to change the sequence design of EXPAR templates in a way that prevents the formation of parasites when dATP is removed from the solution. We further demonstrate that this approach permits the detection of DNA in the presence of contaminating parasites and that it is compatible with the design of bistable reaction networks.

\section{Methods}

Oligonucleotides were purchased from IDT and their sequences displayed in Tables 2 and S1. Template strands were HPLC purified and trigger strands were desalted. The enzymes we used were 8-40 U/mL Bst DNA Polymerase, Large Fragment (NEB), 20-500 U/ml Nb.BssSI (NEB) and 0 or $100 \mathrm{nM}$ of in-house produced thermus thermophilus RecJ exonuclease. ${ }^{20} \mathrm{We}$ noticed a 3.4-fold batch-to-batch change in Nb.BssSI activity. The reaction buffer contained $20 \mathrm{mM}$ Tris- $\mathrm{HCl}, 10 \mathrm{mM}\left(\mathrm{NH}_{4}\right)_{2} \mathrm{SO}_{4}, 50 \mathrm{mM} \mathrm{NaCl}, 1 \mathrm{mM} \mathrm{KCl}, 6 \mathrm{mM} \mathrm{MgSO}$, $1 \mathrm{~g} / \mathrm{L}$ synperonic F 108 (Sigma-Aldrich), 4 mM dithiothreitol, 0.1 g/L BSA (NEB), 1x EvaGreen Dye (Biotium) and 0.1x ROX (invitrogen). Nucleotides (NEB or invitrogen) were added in different concentrations and compositions. In some experiments netropsin was added as indicated. Experiments were performed in a reaction volume of $20 \mu \mathrm{L}$ at $44{ }^{\circ} \mathrm{C}$, with $50 \mathrm{nM}$ template concentration, when necessary, in a CFX96 Touch Real-Time PCR Detection System (Bio-Rad) or a Qiagen Rotor-Gene qPCR machine. The intensity of the green channel was recorded every minute. To avoid cross-contaminations, experiments involving pipetting of solutions containing parasites were performed in a different room using a different set of pipets. 


\section{Results and discussion}

\section{Suppressing untemplated replication}

In EXPAR templated amplification the trigger species $\mathbf{A}$ replicates on template $\mathbf{T}$ with the help of a polymerase and a nickase, noted pol and nick respectively in the following (Figure 1a). There is a series of sequence constrains for the reaction to happen. If we note $a$ the sequence of $\mathbf{A}$, and $\bar{a}$ its complementary, $\mathbf{T}$ is a double repeat of $\bar{a}$, noted $\bar{a} \bar{a}$. In addition, the double stranded species $\overline{\mathbf{T}}: \mathbf{T}$ bears the recognition site of the nickase in such a way that the enzyme cuts $\overline{\mathbf{T}}$ to generate two species $\mathbf{A}$ bound to $\mathbf{T}$ (Figure $1 \mathrm{~b}$ ). As a result, when A binds to the 3' end of $\mathbf{T}$, it is extended by pol to make $\overline{\mathbf{T}}: \mathbf{T}$, which is then cut by nick to form the complex A:T:A. The reaction is isothermal and set up close to the melting temperature of $\mathbf{A}: \mathbf{T}$, here $44^{\circ} \mathrm{C}$, to ensure that $\mathbf{A}$ can dehybridize and take part in reactions with other templates. To close the catalytic loop, either $\mathbf{A}: \mathbf{T}: \mathbf{A}$ dissociates to recycle species $\mathbf{T}$ or it is extended again by pol, which is capable of strand-displacement, regenerating $\overline{\mathbf{T}}: \mathbf{T}$ and producing an extra $\mathbf{A}$.

Untemplated replication in the presence of pol, nick and dNTPs is well documented ${ }^{4,21}$ but its mechanism has not yet been elucidated. ${ }^{22}$ In the current working hypothesis, as soon as a sequence with a hairpin on the 3' appears by de novo synthesis, it may be extended by pol. Subsequent rounds of hairpin formation and slippage followed by polymerization account for the synthesis of palindromic repetitive sequences. Nicking events followed by de novo polymerization of few bases that can self-hybridize may explain the observation of palindromic sequences flanked by nicking sites (Figure 1c).

Figure 1d displays a typical EXPAR experiment where the total concentration of double stranded DNA (dsDNA) is followed by recording the fluorescence of the dsDNA intercalator EvaGreen. A solution containing pol, nick, dNTPs and template strand $\mathbf{T}_{1}$ was incubated at constant temperature (purple line). In the beginning of the reaction, the signal coming from the dye is low since no dsDNA is present. When templated replication occurs, at 18 min, a 
rapid exponential phase leads to a signal increase until the signal saturates, indicating that all template is bound to the trigger. After 200 minutes, a second, non-linear signal increase takes place, which is due to untemplated replication. In the absence of template (yellow line), only the later signal increase is observed. Interestingly, the onset time of untemplated replication is similar in the presence or in the absence of template.

A strategy used so far to mitigate parasite replication was the addition of netropsin, since the parasite sequence was found to be rich in AT repeats by Tan et al. ${ }^{4,19}$ Netropsin helps by binding such AT-rich sequences, but cannot always prevent parasite formation, probably because other studies showed parasites without AT-rich stretches. ${ }^{21}$ Our solution to the problem consists in making it impossible to create secondary structures that can be nicked. Table 1 shows the sequences of the recognition sites for commercially-available nickases. Most of the enzymes, including Nt.BstNBI a common nickase used in EXPAR, contain all four bases (A, C, G, T) in each strand of their recognition site, and thus both templated and untemplated replication need the four dNTPs to proceed. In contrast, Nb.BssSI, Nt.BsmAI and Nt.BspQI have recognition sites with only three bases (C, G, T) on their top strand. As a result, one can design a trigger with only C, G, T and a template with only C, G, A in their sequences. In the absence of dATP, such a system should be able to perform templated replication normally, while being uncapable of untemplated replication. Indeed, if mutations lead to the formation of unwanted products, they might contain secondary structures, but no recognition site for the nickase, which needs two adenines. This unwanted sequence will then only be able to grow by extending the 3'-end, which is not an exponential process and should't interfere too much with autocatalysis. In addition, the absence of dATP in the solution also precludes the formation of AT repeats that are rich in parasites, which might be another reason for reducing the untemplated replication. In the following, we fully characterize our strategy with Nb.BssSI and we demonstrate that it also works with Nt.BsmAI and partially with Nt.BspQI.

Figure 2 demonstrates that this approach works as designed. We incubated template $\mathbf{T}_{1}$ 
Table 1: Top strand sequences for the recognition site of different nicking enzymes (from 5' to 3'). The nicking sites are marked with '. $\mathrm{N}$ means any base. In bold the main enzyme used in this work. In italics the 3 enzymes whose recognition sequence only bears 3 letters on the top strand tested in this study.

\begin{tabular}{ll}
\hline Enzyme & Recognition site \\
\hline $\boldsymbol{N b . B s s \boldsymbol { I }}$ & C'TCGTG \\
Nt.BsmAI & GTCTCN'N \\
Nt.BspQI & GCTCTTCN' \\
Nt.BstNBI & GAGTCNNNN'N \\
Nb.BsmI & NG'CATTC \\
Nb.BsrDI & NN'CATTGC \\
Nb.BtsI & NN'CACTGC \\
Nt.BbvCI & CC'TCAGC \\
Nt.AlwI & GGATCNNNN'N \\
\hline
\end{tabular}

Table 2: DNA sequences for the main set of species used in this work, all working with Nb.BssSI. Asterisks indicate phosphorohioate bonds and $\mathbf{p}$ phosphate modifications.

\begin{tabular}{ll}
\hline Species & Sequence (5’-3') \\
\hline $\mathbf{A}_{1}$ & TCGTGTTCTGTC \\
$\mathbf{T}_{1}$ & G $^{*} \mathrm{~A}^{*} \mathrm{C}^{*}$ AGAAC*ACGAGACAGAACACp \\
$\mathbf{T}_{1}^{u}$ & $\mathrm{G}^{*} \mathrm{~A}^{*} \mathrm{C}^{*}$ AGAACACGAGACAGAACACp \\
$\mathbf{T}_{1}^{3 p}$ & $\mathrm{G}^{*} \mathrm{~A}^{*} \mathrm{C}^{*}$ AGAA* $\mathrm{C}^{*} \mathrm{~A}^{*}$ CGAGACAGAACACp \\
$\mathbf{T}_{1}^{f p}$ & $\mathrm{G}^{*} \mathrm{~A}^{*} \mathrm{C}^{*} \mathrm{~A}^{*} \mathrm{G}^{*} \mathrm{~A}^{*} \mathrm{~A}^{*} \mathrm{C}^{*} \mathrm{~A}^{*} \mathrm{C}^{*} \mathrm{G}^{*} \mathrm{~A}^{*} \mathrm{G}^{*} \mathrm{~A}^{*}-$ \\
& $\mathrm{C}^{*} \mathrm{~A}^{*} \mathrm{G}^{*} \mathrm{~A}^{*} \mathrm{~A}^{*} \mathrm{C}^{*} \mathrm{~A}^{*} \mathrm{C} \mathbf{p}$ \\
$\mathbf{A}_{1} \mathbf{A}_{1}$ & TCGTGTTCTGTCTCGTTTCTGTC \\
$\mathbf{R}_{1}$ & $\mathrm{~A}^{*} \mathrm{~A}^{*} \mathrm{~A}^{*}$ AGACAGAACACGAp \\
\hline
\end{tabular}




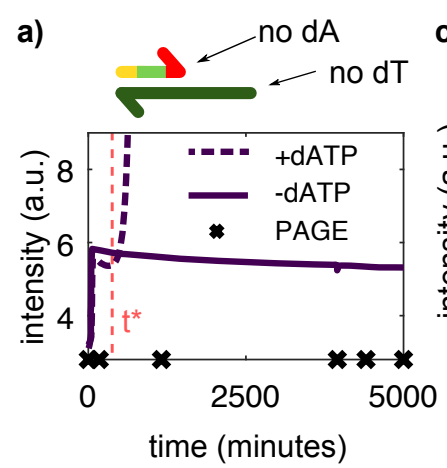

c)

b)

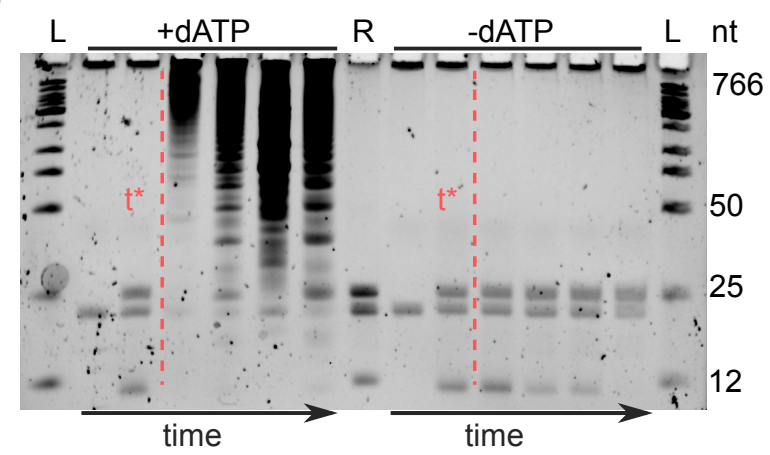

Figure 2: Suppressing dATP blocks untemplated replication without perturbing templated replication. a) Top: We use Nb.BssSI, a nickase that allows to design templates without thymine (dT) and triggers without adenine (dA). Bottom: EvaGreen fluorescence vs. time for an EXPAR reaction of trigger $\mathbf{A}_{1}$ and template $\mathbf{T}_{1}$ in the presence (dashed) and in the absence (solid line) of dATP. Crosses indicate time-points where aliquots were withdrawn for the gel in panel (b). b) Time evolution of the reactions in panel (a) on a PAGE denaturing gel. The red dashed line is a guide to the eye indicating the onset of untemplated replication in the presence of dATP $\left(\mathrm{t}^{*}\right)$. Lanes 4-7 have been diluted 20-fold for easier visualization of parasite bands. $\mathrm{L}$ is a ladder and $\mathrm{R}$ a reference containing species $\mathbf{A}_{1}, \mathbf{T}_{1}$ and $\mathbf{A}_{1} \mathbf{A}_{1}$. c) EXPAR reactions for three different sequences working with nickase Nb.BssSI and one sequence working with Nt.BsmAI in the presence (dashed) and in the absence (solid line) of dATP. Conditions: 8 (a,b) or 4.8 (c) U/mL pol, $20 \mathrm{U} / \mathrm{mL}$ nick, 1 (a,b) or 0.4 (c) mM dNTPs. exo is $100 \mathrm{nM}$ in panels a and b and in Nt.BsmAI reactions in panel c, and $0 \mathrm{nM}$ in Nb.BssSI reactions in panel c.

with pol and nick in the presence or in the absence of dATP. Because we will later use this system to design more complex molecular programs, template $\mathbf{T}_{1}$ lacked two bases in the 3' side compared to the complementary of a double repeat of the trigger sequence $\mathbf{A}_{1}$, i.e. $\mathbf{A}_{1}$ was a 12 -mer, $\mathbf{T}_{1}$ a 22 -mer and when $\mathbf{A}_{1}$ binds to $\mathbf{T}_{1}$ and is extended by the polymerase, the 24-mer $\mathbf{A}_{1} \mathbf{A}_{1}$ is formed (Table 2). In addition, ttRecJ, a single-stranded specific exonuclease with 5' activity, noted exo, was added to the reaction to ensure that template replication was 
active throughout the duration of the experiment. $\mathbf{T}_{1}$ was protected from the exonuclease by 3 phosphorothioate (PTO) bonds at its 5' end, and bear a fourth one in the middle of the sequence to protect against background restriction activity (see below). In these conditions, when dATP was present, both templated and untemplated replication were observed, while only templated amplification was observed in the absence of dATP (Figure 2a). Analysis of the reaction products on a denaturating polyacrylamide gel confirmed that at long times, large amounts of strands much longer than the trigger, which we identify with the parasite, only form when dATP is present (Figure 2b). Before the emergence of the parasite, the reaction products were indistinguishable both in the presence or in the absence of dATP: 3 bands at 12, 22 and 24-nt corresponding respectively to $\mathbf{A}_{1}, \mathbf{T}_{1}$ and $\mathbf{A}_{1} \mathbf{A}_{1}$. After 200 min — when the parasite has already appeared in the presence of dATP - no new bands appear in the -dATP sample. Finally, parasite suppression is compatible with different template sequences (SI Table S1) and the three nickases in Table 1 suitable to our threeletter approach. Figure 2c shows three sets of sequences working with Nb.BssSI and one set working with Nt.BsmAI that displayed templated but not untemplated replication in the absence of dATP. In our experimental conditions, parasite suppression worked with Nt.BspQI but not templated amplification, probably because this enzyme has low efficiency in the buffer used here (SI Figure S1). The experiments with Nb.BssSI in Figure 2c were

performed in the absence of exo to demonstrate that degradation is not needed for parasite suppression. Because this approach allows to perform EXPAR reactions that are robust against untemplated replication we will call it rEXPAR in the following.

\section{Nb.BssSI nickase displays a background restriction activity that can be easily suppressed}

Nb.BssSI, the nickase used here has been recently developed and has seldom been used in EXPAR experiments to our knowledge. In our preliminary experiments we used template $\mathbf{T}_{1}^{u}$, identical to $\mathbf{T}_{1}$ except for a missing PTO after the 8 th nucleotide, and we observed 
a)

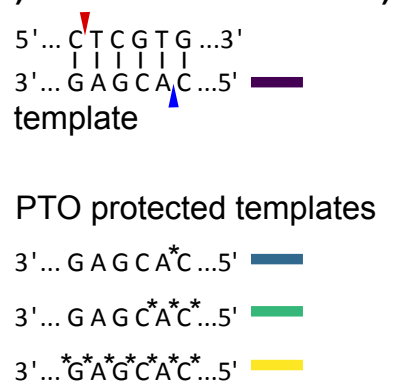

b)

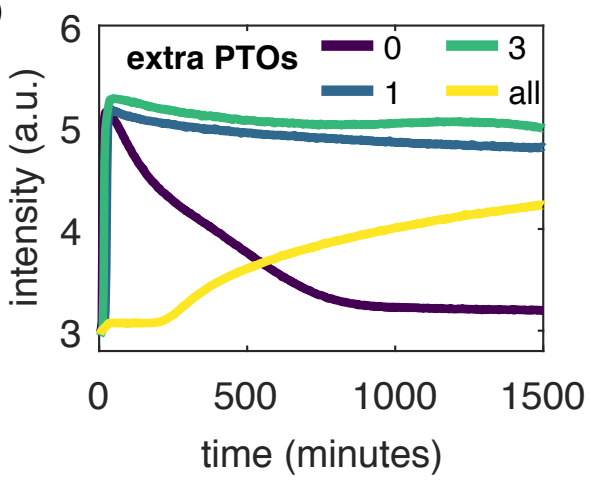

Figure 3: Nb.BssSI background restriction activity can be suppressed by adding a PTO bond to the template strand. a) The nickase Nb.BssSI is supposed to cut at the site indicated in red. The restriction enzyme BssSI additionally cuts at the site indicated in blue. We tested four templates, $\mathbf{T}_{1}^{u}$ (purple), $\mathbf{T}_{1}$ (blue), $\mathbf{T}_{1}^{3 p}$ (green), $\mathbf{T}_{1}^{f p}$ (yellow) with increasing number of PTOs around the BssSI site. b) EvaGreen fluorescence vs. time for rEXPAR experiments with templates in (a). Conditions: $8 \mathrm{U} / \mathrm{mL}$ pol, $200 \mathrm{U} / \mathrm{mL}$ nick, $100 \mathrm{nM}$ exo, $0.4 \mathrm{mM}$ dNTPs.

a significant loss of fluorescence signal in many long-term experiments (Figure 3b purple line). In EXPAR experiments with other nicking enzymes (Nb.BsmI, Nt.BstNBI) we did not observe this behavior. With $\mathbf{T}_{1}^{u}$, increasing Nb.BssSI concentrations promoted longterm signal loss while the addition of $\mathbf{T}_{1}^{u}$ temporarily restored the signal (SI Figure S2). We hypothesized that, because Nb.BssSI was derived from the restriction enzyme BssSI, it may have a reminiscent restriction activity, which would cleave the template strand. To solve this problem, we tested different templates with additional PTO protection (note that all 5'-ends have 3 PTOs for protection from exonuclease). Figure 3b shows EXPAR experiments with the protected templates. The unprotected template $\mathbf{T}_{1}^{u}$ shows degradation after only $20 \mathrm{~min}$. The fully protected template $\mathbf{T}_{1}^{f p}$ does not only have the PTOs in the recognition sequence, but between all 22 bases. This leads to a strong inhibition of templated replication that could arise from the higher melting temperature of PTO-modified strands or from the inhibition of pol or nick. In contrast, templates with $1, \mathbf{T}_{1}$, or $3, \mathbf{T}_{1}^{3 p}$, PTOs surrounding the putative second nicking site associated to the BssSI activity on the template strand show almost uninhibited replication and a stable signal in the steady state for at least 5000 minutes (Figure 2a). 


\section{Standard methods delay parasite emergence but do not suppress it}

Standard methods to mitigate untemplated replication involve decreasing pol or increasing nick concentrations ${ }^{9}$ and adding netropsin. ${ }^{4,19}$ Figure 4 shows that these approaches delay but do not suppress the onset of parasite emergence. In the absence of template, the parasite onset time, $\tau_{u}$, is inversely proportional to pol concentration, as expected from first-order kinetics (Figure 4a,b). In contrast, increasing nick concentration increases $\tau_{u}$, specially at low pol concentration (SI Figure S3), suggesting that nick inhibits pol. Finally, adding up to $4 \mu \mathrm{M}$ netropsin delays parasite emergence 4-fold but it also slows down templated replication by the same amount and reduces the fluorescence signal from the dsDNA intercalator dye (Figure 4c and SI Figure S4). In summary, all these methods slow down untemplated replication at the cost of slowing down templated replication as well, which is an undesirable feature for rapid analysis.

\section{rEXPAR is compatible with PEN molecular programs}

If a chemical reaction is monostable in the sense of dynamical systems, the involved species reach a single steady state concentration independently of the initial conditions. Because the EXPAR autocatalytic network is intrinsically monostable, in the absence of trigger strand (i.e. $A=0$ ), an infinitesimally small addition of $\mathbf{A}$ will grow exponentially until reaching the steady state. This is problematic to detect very low amounts of $\mathbf{A}$, as any unprimed synthesis of $\mathbf{A}$ will result in an undesired background, known as early stage background amplification or self-start. One can make EXPAR robust to self-start by using instead a bistable autocatalytic network based on the polymerase, nickase, exonuclease dynamic network assembly toolbox (PEN DNA toolbox). ${ }^{10}$

The PEN DNA toolbox is an experimental framework based on the EXPAR reaction that allows designing reaction networks that mimick the dynamics of gene regulatory networks

in solution. ${ }^{6,19,23}$ This framework makes network design straightforward because network topology is defined by predictable interactions between short ssDNAs. In addition, the com- 
a)

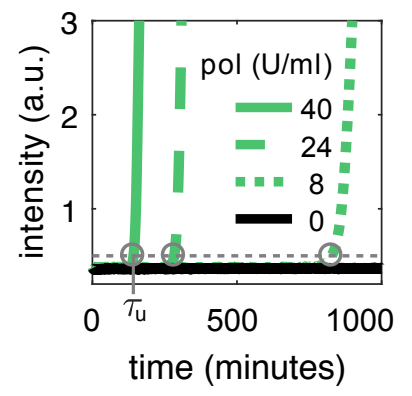

b)

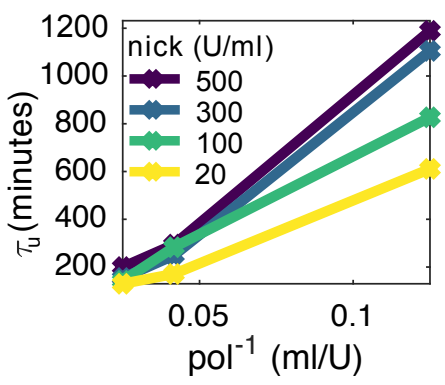

c)

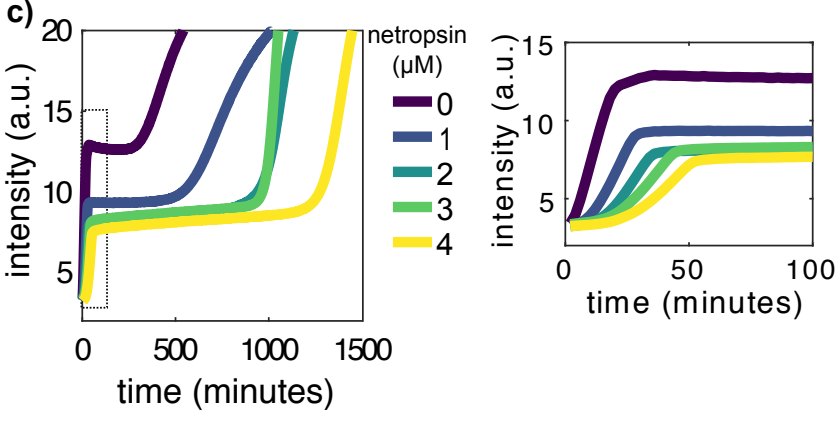

Figure 4: Standard approaches delay untemplated replication but do not suppress it. a) EvaGreen fluorescence $v s$. time in the absence of $\mathbf{T}_{1}$ for different polymerase (pol) concentrations at $100 \mathrm{U} / \mathrm{ml}$ nickase. Circles indicate the onset time of untemplated replication $\tau_{u}$. b) $\tau_{u}$ vs. the inverse of pol concentration for different nick concentrations. c) EvaGreen fluorescence $v s$. time in the presence of $\mathbf{T}_{1}$ for different netropsin concentrations. The plot on the right is a zoom of the data on the left inside the dotted rectangle. Conditions: 0.4 $\mathrm{mM}$ dNTPs, $100 \mathrm{nM}$ exo (for a-b) and $8 \mathrm{U} / \mathrm{mL}$ pol, $60 \mathrm{U} / \mathrm{mL}$ nick (for c).

bination of its three core enzymes with large quantities of dNTPs provides a convenient way to keep the network out of equilibrium in a closed reactor for very long time at steady state. These two unmatched properties have allowed the rational design of complex spatio-temporal behaviors such as oscillations, ${ }^{6,24}$ bistability ${ }^{7,10}$ and reaction-diffusion patterns. ${ }^{8,25-27}$ Besides suppressing background amplification in EXPAR, such dynamic behaviors have important applications such as in nucleic acid detection, ${ }^{28}$ in material science, ${ }^{9}$ in the design of microrobots $^{29}$ and in protein directed evolution. ${ }^{30}$ However, untemplated amplification is an important obstacle for these applications because it precludes the use of PEN circuits for long periods of time.

PEN networks are usually built around one or more autocatalytic nodes based on EXPAR. 
An exonuclease enzyme is added such that nodes are not only dynamically produced but also degraded. In addition to the autocatalytic template strands intrinsic to EXPAR, that catalyze the reaction $\mathbf{A} \rightarrow 2 \mathbf{A}$, other templates can be used to catalyze other processes such as activation $(\mathbf{A} \rightarrow \mathbf{A}+\mathbf{B})$, and repression $(\mathbf{A} \rightarrow \emptyset)$. All these template strands bear PTOs in 5' to protect them from exonuclease degradation.

a)
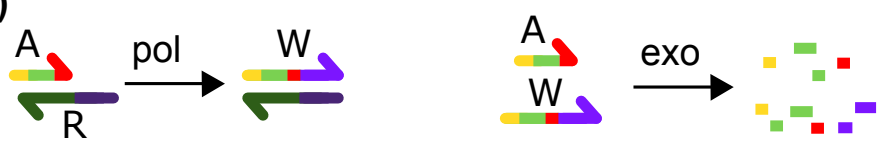

b)

c)
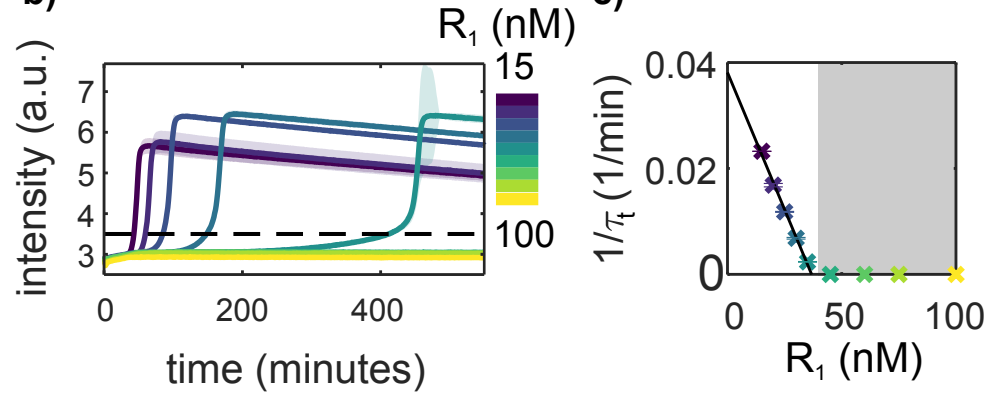

Figure 5: rEXPAR is compatible with PEN DNA bistable programs. a) In addition to the autocatalytic network depicted in Fig. 1a, a repressor template $\mathbf{R}$ is added that binds to trigger $\mathbf{A}$ and turns it into waste $\mathbf{W}$, which can be degraded by exonuclease but cannot prime autocatalysis. b) EvaGreen fluorescence vs. time in the presence of $\mathbf{T}_{1}^{u}$ but in the absence of $\mathbf{A}_{1}$, for increasing concentrations of $\mathbf{R}_{1}$. The dashed line indicates the threshold corresponding to the onset time of templated amplification $\tau_{t}$. c) $1 / \tau_{t}$ vs. $\mathbf{R}$ concentration from panel (b). The black line corresponds to a linear fit of slope $1.0 \times 10^{-3} \mathrm{nM}^{-1} \mathrm{~min}^{-1}$. The gray area indicates where the system is bistable and thus robust to self-start. Experiments performed in the absence of dATP. The shade and the error bars in panels $\mathrm{b}$ and $\mathrm{c}$ correspond to the standard deviation of a triplicate experiment. Conditions: $8 \mathrm{U} / \mathrm{mL}$ pol, $20 \mathrm{u} / \mathrm{mL}$ nick, $100 \mathrm{nM}$ exo and $0.4 \mathrm{mM}$ dNTPs.

In this framework, to render bistable an EXPAR reaction, and thus suppress self-start, one just needs to add a second template strand $\mathbf{R}$ that binds to trigger $\mathbf{A}$ and, with the help of pol, it extends it into the waste strand $\mathbf{W}$, that can be degraded by exo but not recognized by nick (Figure 5a). In addition, the polymerization reaction of $\mathbf{A}$ on $\mathbf{R}$ needs to be faster than the one of $\mathbf{A}$ on its template $\mathbf{T}$. To fulfill these two requirements, we chose to use a repressor strand $\mathbf{R}_{1}$ with a sequence of 4 adenines followed by the reverse complementary 
sequence of $\mathbf{A}_{1}$ (Table 2) and a template strand that lacks on the 3' end two bases to be fully complementary to $\mathbf{A}_{1}$, here $\mathbf{T}_{1}^{u} \cdot \mathbf{R}_{1}$ was protected against exonuclease degradation by 3 PTO bonds in the 5' end. We incubated $\mathbf{T}_{1}^{u}$ in the presence of pol, nick and exo and increasing concentrations of $\mathbf{R}_{1}$, noted $R_{1}$, in the absence of trigger $\mathbf{A}_{1}$ and dATP. At the lowest $R_{1}$ tested of $15 \mathrm{nM}$, untriggered templated amplification was observed within $30 \mathrm{~min}$, indicating that the system is monostable with a single stable point at high $\mathbf{A}_{1}$ concentration. Increasing $R_{1}$ resulted in a dramatic increase of the templated amplification time $\tau_{t}$ until it became undetectable (> $1000 \mathrm{~min}$, SI Figure S6) above $R_{1}=60 \mathrm{nM}$ (Fig. 5b-c). Above this threshold the reaction network becomes bistable with a stable point at $A_{1}=0$ and a second one at high $A_{1}$. A plot of $1 / \tau_{t}$ as a function of the concentration of $\mathbf{R}_{1}$ indicates that $R_{1}$ is a bifurcation parameter of the reaction network. Because dATP was absent, no untemplated amplification was observed in the system, demonstrating that rEXPAR is compatible with the construction of DNA-based reaction networks with complex dynamics, such as bistability.

\section{rEXPAR is robust to parasite contamination}

Besides being an intriguing instance of molecular evolution, parasites can easily contaminate the laboratory ${ }^{31}$ and produce false positives in EXPAR because they amplify as fast as target DNA and produce a higher fluorescent signal. Figure 6 shows EXPAR amplification experiments in the presence and in the absence of dATP (rEXPAR) for samples with or without parasite contamination. Contamination consisted in a 3000-fold dilution of another sample that had previously undergone untemplated amplification. This amount corresponds to using a pipet tip that has been filled and emptied with $1 \mu \mathrm{L}$ of a solution containing parasite (SI Figure S5). We observed that templated and untemplated amplification occurred concomitantly in the sample containing both dATP and parasite. In contrast, rEXPAR samples without dATP were robust to untemplated amplification both in the absence and in the presence of contamination. 

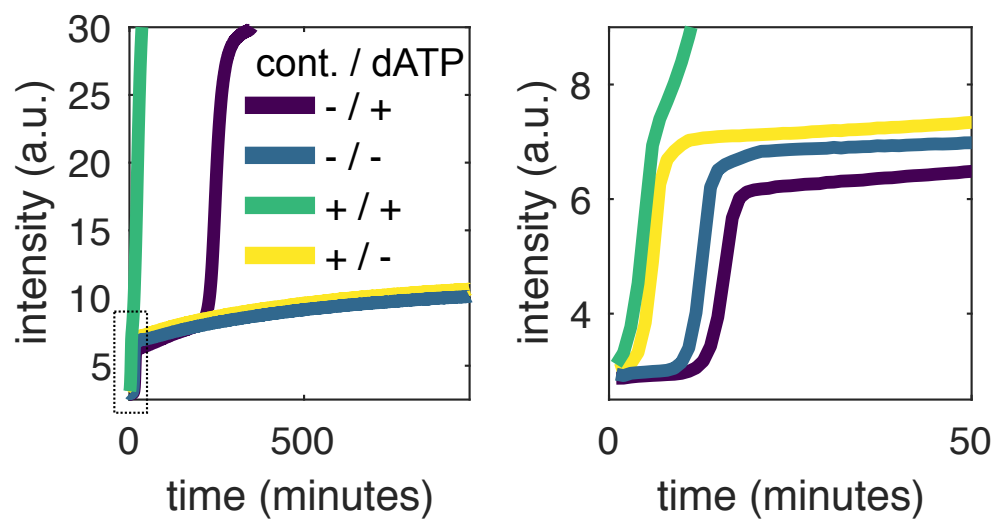

Figure 6: rEXPAR is robust to parasite contamination. EvaGreen fluorescence vs. time for EXPAR experiments performed both with and without dATP and with and without contamination from a parasite solution diluted 3000-fold (cont.). The plot on the right is a zoom of the data on the left inside the dotted rectangle. Conditions: $40 \mathrm{U} / \mathrm{mL}$ po, 500 $\mathrm{U} / \mathrm{mL}$ nick, $0 \mathrm{nM}$ exo, $60 \mathrm{nM} \mathbf{R}_{1}, 0.4 \mathrm{mM}$ dNTPs.

In a second set of experiments (Figure 7 and SI Figure S9) we evaluated the performance of EXPAR and rEXPAR to detect trigger DNA in the presence of parasite contamination. To be more realistic about a fortuitous parasite contamination we used a higher dilution of $10^{6}$-fold. EXPAR and rEXPAR attained a similar limit of detection of $0.4 \mathrm{pM}$ in the absence of contamination, although rEXPAR was 1.6-fold faster (SI Figures S7-8). In contrast, in the presence of contamination, rEXPAR was able to detect the trigger without noticeable change while EXPAR was unable to detect even the highest trigger concentration of 100 pM.

\section{Conclusion}

We took advantage of a new nicking enzyme, Nb.BssSI, whose recognition site has only a three-letter code on the top strand (C'TCGTG), to perform EXPAR isothermal amplification experiments in the absence of one deoxynucleotide (dATP). In these conditions, templated amplification proceeded normally and even slightly faster, while untemplated amplification, resulting in the production of an autocatalytic set of parasitic sequences, was completely suppressed. Our approach, called rEXPAR for robust EXPAR, contrasts with existing methods, such as the addition of netropsin, that mitigate but do not suppress untemplated amplifi- 

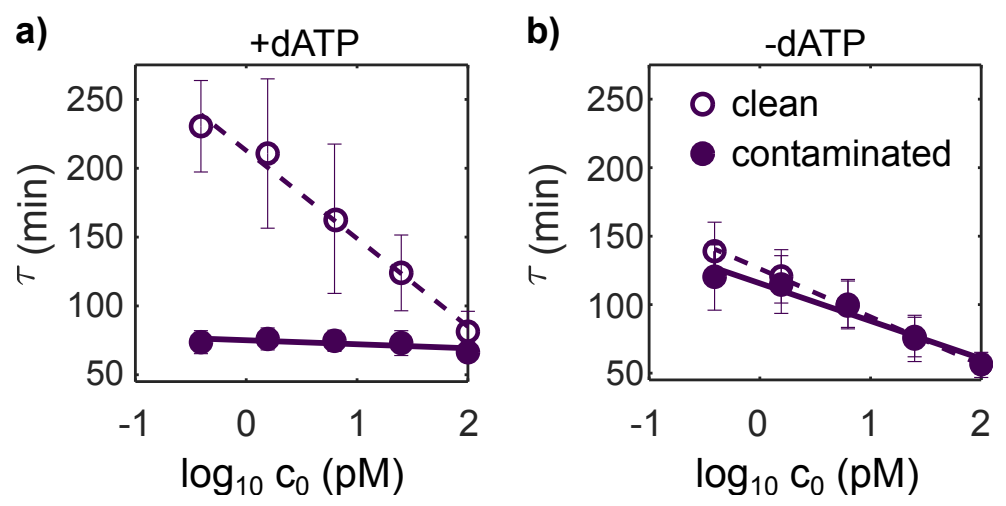

Figure 7: rEXPAR is able to detect trigger DNA in the presence of parasite contamination, in contrast with EXPAR. Amplification onset time, $\tau$, vs. the decimal logarithm of the initial trigger concentration $c_{0}$ for EXPAR reactions performed in presence (a) and in the absence (b) of dATP, with (filled symbols) and without (empty symbols) contamination from a parasite solution diluted $10^{6}$-fold. Error bars correspond to the standard deviation from three different experiments performed at different days with different stock solutions. Conditions: $8 \mathrm{U} / \mathrm{mL}$ pol, $20 \mathrm{U} / \mathrm{mL}$ nick, $100 \mathrm{nM}$ exo, $15 \mathrm{nM} \mathbf{R}_{1}, 0.4 \mathrm{mM}$ dNTPs.

cation. rEXPAR is also compatible with other 3-letter nicking enzymes, such as Nt.BsmAI. rEXPAR is compatible with EXPAR-based molecular programming languages such as the PEN DNA toolbox, which we demonstrated by implementing a bistable autocatalytic network that suppressed self-start spurious reactions. In addition rEXPAR, in contrast with EXPAR, allows the detection of trigger DNA in the presence of minute amounts of parasite contamination. As a result, we believe that rEXPAR will be useful, both for running out-ofequilibrium molecular programs over extended periods of time, which is essential for building 'life-like" materials, ${ }^{9}$ and for making EXPAR more robust in analytical applications.

\section{Supporting Information Available}

The supplementary data contains extra DNA sequences, characterization of the background restriction activity of $\mathrm{Nb.BssSI}$, quantification of netropsin effect on kinetics, parasite replication kinetics, effect of dNTPs concentration on kinetics and amplification curves corresponding to Fig. 7. This material is available free of charge via the Internet at http: //pubs.acs.org/. 


\section{Acknowledgement}

Yannick Rondelez and Guillaume Ginés for insightful discussions.

\section{Funding}

This work has been funded by the European Research Council (ERC) under the European's Union Horizon 2020 programme (grant No 770940, A.E.-T.), by the Deutsche Forschungsgemeinschaft (grant No 364775124, G.U.), by the Ville de Paris Emergences programme (Morphoart, A.E.-T.), by a Marie Sklodowska-Curie fellowship (grant No 795580, M.V.D.H.) from the European Union's Horizon 2020 programme and by a PRESTIGE grant (grant No 609102, M.V.D.H.) from the European Union's Seventh Framework Programme.

\section{References}

(1) Zhao, Y.; Chen, F.; Li, Q.; Wang, L.; Fan, C. Isothermal Amplification of Nucleic Acids. Chem. Rev. 2015, 115, 12491-12545.

(2) Reid, M. S.; Le, X. C.; Zhang, H. Exponential isothermal amplification of nucleic acids and amplified assays for proteins, cells, and enzyme activities. Angewandte Chemie International Edition 2018, 57, 11856-11866.

(3) Van Ness, J.; Van Ness, L. K.; Galas, D. J. Isothermal reactions for the amplification of oligonucleotides. Proc. Nat. Acad. Sci. USA 2003, 100, 4504-4509.

(4) Tan, E.; Erwin, B.; Dames, S.; Ferguson, T.; Buechel, M.; Irvine, B.; Voelkerding, K.; Niemz, A. Specific versus nonspecific isothermal DNA amplification through thermophilic polymerase and nicking enzyme activities. Biochemistry 2008, 47, 9987-9999.

(5) Zhang, Y.; Hu, J.; Zhang, C.-y. Sensitive Detection of Transcription Factors by Isother- 
mal Exponential Amplification-Based Colorimetric Assay. Anal. Chem. 2012, 84, 95449549.

(6) Montagne, K.; Plasson, R.; Sakai, Y.; Fujii, T.; Rondelez, Y. Programming an in vitro DNA oscillator using a molecular networking strategy. Mol. Sys. Biol. 2011, 7, 466.

(7) Padirac, A.; Fujii, T.; Rondelez, Y. Bottom-up construction of in vitro switchable memories. Proc. Nat. Acad. Sci. USA 2012, 109, E3212-E3220.

(8) Padirac, A.; Fujii, T.; Estévez-Torres, A.; Rondelez, Y. Spatial waves in synthetic biochemical networks. J. Am. Chem. Soc. 2013, 135, 14586-14592.

(9) Zadorin, A. S.; Rondelez, Y.; Gines, G.; Dilhas, V.; Urtel, G.; Zambrano, A.; Galas, J. C.; Estevez-Torres, A. Synthesis and materialization of a reaction-diffusion French flag pattern. Nature Chem. 2017, 9, 990-996.

(10) Montagne, K.; Gines, G.; Fujii, T.; Rondelez, Y.; Fukuyama, K. Boosting functionality of synthetic DNA circuits with tailored deactivation. Nature Comm. 2016, \%, 13474.

(11) Chen, J.; Zhou, X.; Ma, Y.; Lin, X.; Dai, Z.; Zou, X. Asymmetric exponential amplification reaction on a toehold/biotin featured template: an ultrasensitive and specific strategy for isothermal microRNAs analysis. Nucleic Acids Res. 2016, 44, e130.

(12) Reid, M. S.; Paliwoda, R. E.; Zhang, H.; Le, X. C. Reduction of Background Generated from Template-Template Hybridizations in the Exponential Amplification Reaction. Anal. Chem. 2018, 90, 11033-11039.

(13) Mills, D. R.; Peterson, R. L.; Spiegelman, S. An extracellular Darwinian experiment with a self-duplicating nucleic acid molecule. Proc. Nat. Acad. Sci. USA 1967, 58, $217-224$. 
(14) Liang, X.; Jensen, K.; Frank-Kamenetskii, M. D. Very Efficient Template/PrimerIndependent DNA Synthesis by Thermophilic DNA Polymerase in the Presence of a Thermophilic Restriction Endonuclease. Biochemistry 2004, 43, 13459-13466.

(15) Kaboev, O. K.; Luchkina, L. A. Template-Free Primer-Independent DNA Synthesis by Bacterial DNA Polymerases I Using the DnaB Protein from Escherichia coli. Dokl. Biochem. Biophys. 2004, 398, 265-267.

(16) Emery, N. J.; Majumder, S.; Liu, A. P. Synergistic and non-specific nucleic acid production by T7 RNA polymerase and Bsu DNA polymerase catalyzed by single-stranded polynucleotides. Syst. Synth. Biol. 2018, 3, 130-134.

(17) Brownie, J.; Shawcross, S.; Theaker, J.; Whitcombe, D.; Ferrie, R.; Newton, C.; Little, S. The elimination of primer-dimer accumulation in PCR. Nucleic Acids Res. 1997, 25, 3235-3241.

(18) Urtel, G. C.; Rind, T.; Braun, D. Reversible Switching of Cooperating Replicators. Phys. Rev. Lett. 2017, 118, 1-5.

(19) Baccouche, A.; Montagne, K.; Padirac, A.; Fujii, T.; Rondelez, Y. Dynamic DNAtoolbox reaction circuits: A walkthrough. Methods 2014, 67, 234-249.

(20) Wakamatsu, T.; Kitamura, Y.; Kotera, Y.; Nakagawa, N.; Kuramitsu, S.; Masui, R. Structure of RecJ exonuclease defines its specificity for single-stranded DNA. J. Biol. Chem. 2010, 285, 9762-9.

(21) Zyrina, N. V.; Zheleznaya, L. A.; Dvoretsky, E. V.; Vasiliev, V. D.; Chernov, A.; Matvienko, N. I. N.BspD6I DNA nickase strongly stimulates template-independent synthesis of non-palindromic repetitive DNA by Bst DNA polymerase. Biol. Chem. 2007, 388, 367-372. 
(22) Zyrina, N. V.; Antipova, V. N.; Zheleznaya, L. A. Ab initio synthesis by DNA polymerases. FEMS Microbiol. Lett. 2014, 351, 1-1.

(23) Padirac, A.; Fujii, T.; Rondelez, Y. Nucleic acids for the rational design of reaction circuits. Curr. Op. Biotech. 2013, 24, 575 - 580, Nanobiotechnology • Systems biology.

(24) Fujii, T.; Rondelez, Y. Predator - Prey molecular ecosystems. ACS Nano 2013, 7, $27-34$.

(25) Zadorin, A. S.; Rondelez, Y.; Galas, J.-C.; Estevez-Torres, A. Synthesis of programmable reaction-diffusion fronts using DNA catalyzers. Phys. Rev. Lett. 2015, 114, 068301.

(26) Zambrano, A.; Zadorin, A. S.; Rondelez, Y.; Estévez-Torres, A.; Galas, J. C. Pursuitand-Evasion Reaction-Diffusion Waves in Microreactors with Tailored Geometry. J. Phys. Chem. B 2015, 119, 5349-5355.

(27) Kurylo, I.; Gines, G.; Rondelez, Y.; Coffinier, Y.; Vlandas, A. Spatiotemporal control of DNA-based chemical reaction network via electrochemical activation in microfluidics. Scientific Reports 2018, 8, 6396.

(28) Rondelez, Y.; Gines, G.; Montagne, K.; Fujii, T. Method of eliminating background amplification of nucleic acid targets. 2016; PCT/IB2016/000352.

(29) Gines, G.; Zadorin, A. S.; Galas, J.-C.; Fujii, T.; Estevez-Torres, A.; Rondelez, Y. Microscopic agents programmed by DNA circuits. Nature Nanotech. 2017, 12, 351359.

(30) Drame-Maigné, A. Compartmentalized directed evolution of enzymes using molecular programs as self-selection function. PhD thesis, Université Paris Descartes, 2018.

(31) Marshall, K. A.; Ellington, A. D. Molecular Parasites That Evolve Longer Genomes. J Mol. Evol. 1999, 49, 656-663. 


\title{
Supplementary information for:
}

\section{rEXPAR: an isothermal amplification scheme that is robust to autocatalytic parasites}

\author{
Georg Urtel, ${ }^{\dagger, \ddagger}$ Marc Van Der Hofstadt, \\ Estevez-Torres, ${ }^{*}, \ddagger$ \\ †Sorbonne Université, Laboratoire Jean Perrin, F-75005, Paris, France \\ $\ddagger U M R$ 8237, CNRS, F-75005, Paris, France \\ E-mail: andre.estevez-torres@upmc.fr
}




\section{Contents}

1 Sequences $\quad$ S3

2 Parasite suppression works for the three compatible nicking enzymes S4

3 Nb.BssSI degrades unprotected templates $\quad$ S5

4 Parasite emergence depends on enzyme concentrations $\quad$ S6

$5 \quad$ Netropsin slows down templated replication $\quad$ S7

6 Parasite replication kinetics $\quad$ S8

$\begin{array}{llr}7 & \text { Bistability } & \text { S10 }\end{array}$

8 Increasing nucleotide concentration slows down templated amplification S11

9 Amplification curves for EXPAR and rEXPAR in the presence of parasite contamination 


\section{Sequences}

Additional oligonucleotide sequences that do not appear in Table 2 in the MT can be found in Table S1. DNA was purchased from Integrated DNA Technologies, Inc.

Table S1: DNA sequences with modifications. PTOs are marked with an asterisk * and phosphates with $\mathbf{p}$.

\begin{tabular}{|c|c|c|}
\hline Name & Sequences $5^{\prime} \rightarrow 3$ ' & Nickase \\
\hline $\mathrm{T}_{1}^{+2}$ & $\mathrm{G}^{*} \mathrm{~A}^{*} \mathrm{C}^{*}$ AGAACACGAGACAGAACACGAp & Nb.BssSI \\
\hline $\mathrm{A}_{2}$ & TCGTGGTTCTTC & Nb.BssSI \\
\hline $\mathrm{T}_{2}$ & $\mathrm{G}^{*} \mathrm{~A}^{*} \mathrm{~A}^{*}$ GAACCACGAGAAGAACCACp & Nb.BssSI \\
\hline $\mathrm{A}_{3}$ & TCGTGTCTGTC & Nb.BssSI \\
\hline $\mathrm{T}_{3}$ & G*A*C*AGACACGAGACAGACACGp & Nb.BssSI \\
\hline $\mathrm{T}_{4}$ & $\mathrm{~A}^{*} \mathrm{G}^{*} \mathrm{~A}^{*} \mathrm{GACAGAACCAGAGACAGAAp}$ & Nt.BsmAI \\
\hline $\mathrm{A}_{4}$ & GGTTCTGTCTCT & Nt.BsmAI \\
\hline $\mathrm{T}_{5}$ & $\mathrm{~A}^{*} \mathrm{G}^{*} \mathrm{~A}^{*}$ AGGACGAGAAGAAGGACGAp & Nt.BspQI \\
\hline $\mathrm{A}_{5}$ & TCTCGTCCTTCT & Nt.BspQI \\
\hline
\end{tabular}




\section{Parasite suppression works for the three compatible nicking enzymes}

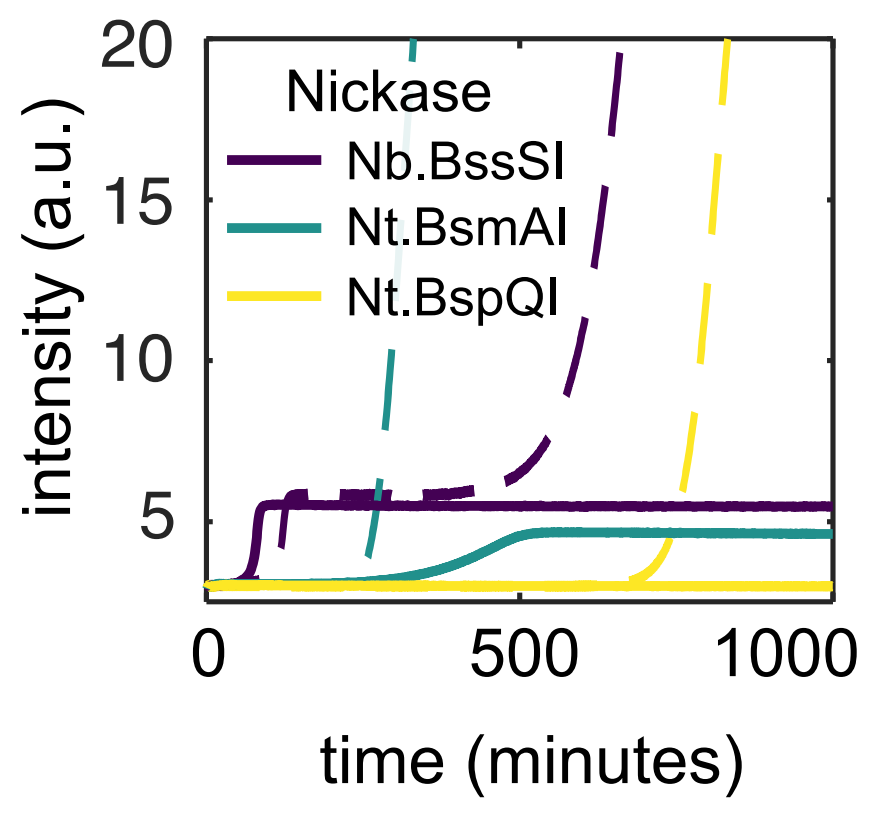

Figure S1: EXPAR reactions for three different nickases in the presence (dashed) and in the absence (solid line) of dATP. For all the nickases, suppressing dATP suppressed the parasite. However, in our experimental conditions only Nb.BssSI and Nt.BsmAI produced templateamplified EXPAR while Nt.BspQI did not (solid yellow line is flat), probably because it has low efficiency in the working buffer. 


\section{$3 \quad$ Nb.BssSI degrades unprotected templates}

During preliminary experiments, we observed a loss of fluorescence signal in many of the long-term experiments, for example in Figure S2a. Two possible reasons for this signal loss are template degradation by Nb.BssSI or a degradation of Nb.BssSI itself. Since the signal loss is stronger for higher concentrations of nickase, a template degradation seemed more likely. To test this assumption, we injected either template or Nb.BssSI to the samples which showed the strongest degradation (Fig. S2b). In addition, the storage medium of template (water) or Nb.BssSI (diluent B, NEB) was injected with the same volumes as a control to compensate for dilution effects. Only the injection of template restores the signal, which starts to slowly decrease again.
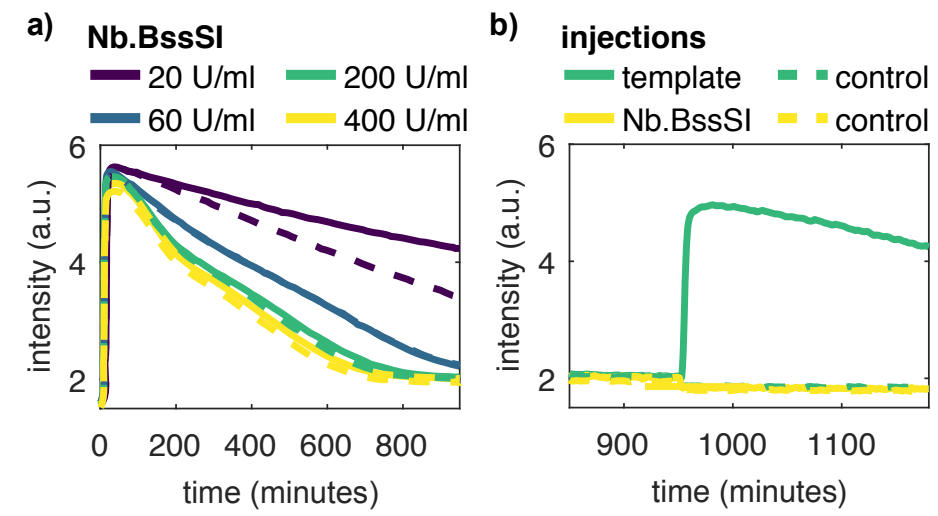

Figure S2: Degradation of templates by Nb.BssSI. a) Autocatalysis with a range of nicking enzyme shows a signal loss over time. Two samples were used for each nickase concentration (solid, dashed). b) For the samples with the strongest signal loss in panel (a), injections were performed. Only injecting template restores the signal. Nickase injections or controls with the respective buffer show no effect. 


\section{Parasite emergence depends on enzyme concentra- tions}

As described in the main text, the emergence of parasites depends on the concentrations of nickase and polymerase as well as the presence of all four dNTPs. Figure S3 show the data represented in Figure 4a-b of the main text plotted in different ways to highlight the effect of nick concentration.
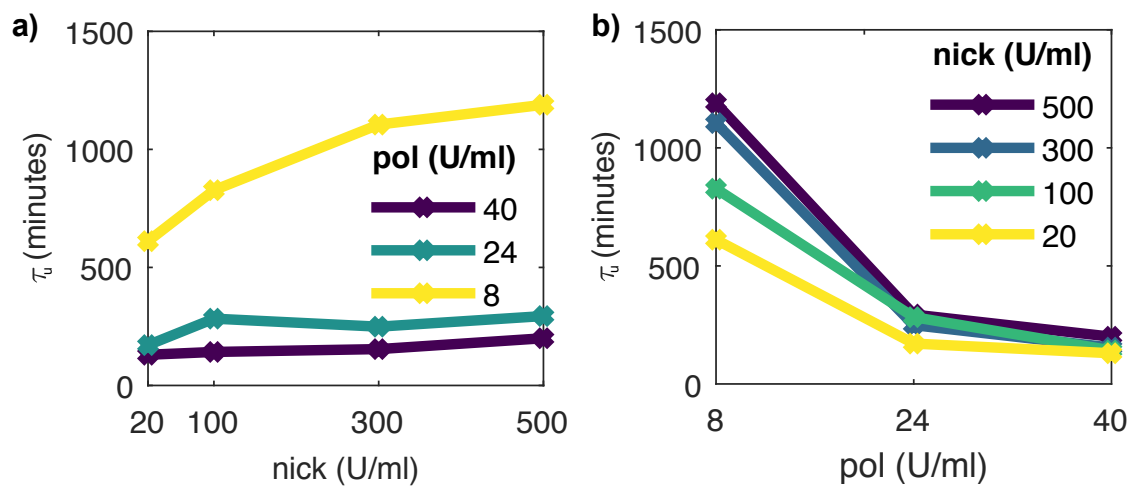

Figure S3: Onset time of untemplated replication $\tau_{u}$ for different polymerase and nickase concentrations. 


\section{Netropsin slows down templated replication}

In Figure 4c of the main text it was shown that netropsin helps to delay the emergence of parasite. Netropsin also has some side effects. It decreases the fluorescence intensity of EvaGreen (Fig. S4a) and slows down the reaction rate (Fig. S4b-d). We found that the rate is proportional to $1 /[$ netropsin]. Comparing the rates for the sample with $4 \mu \mathrm{M}$ netropsin to the control sample without netropsin, shows a 4.5-fold decrease in templated replication rate.
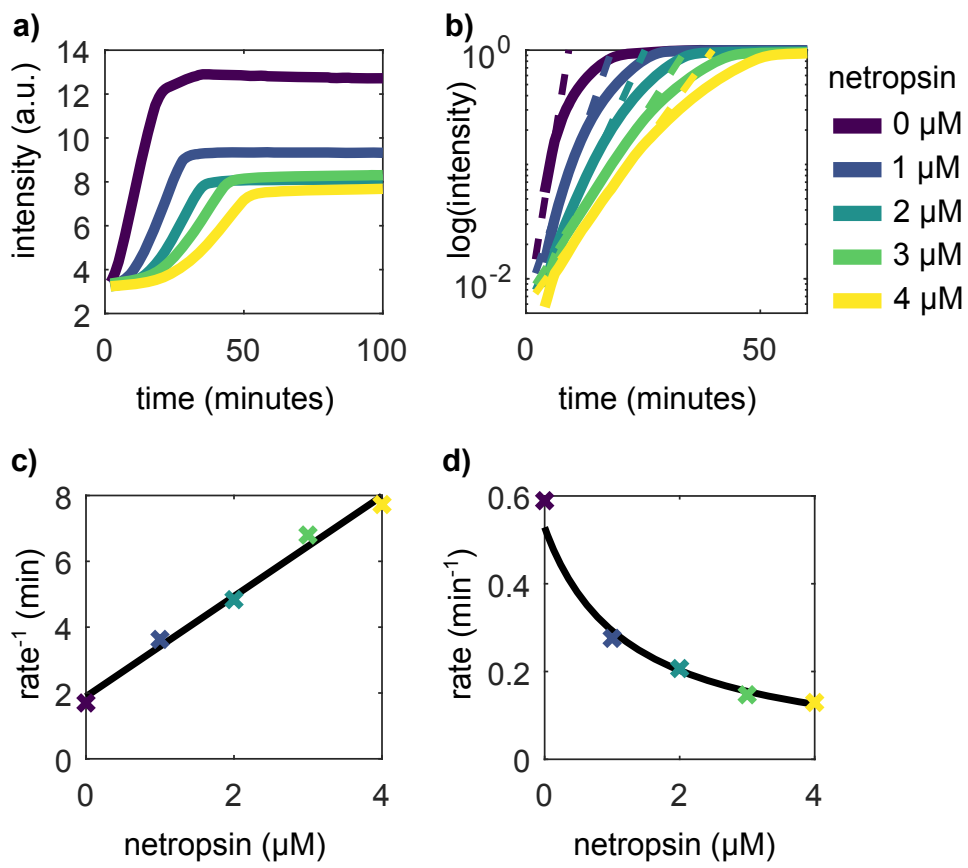

Figure S4: Netropsin inhibits templated replication. The legend for all four plots is on the right side. a) This is the inset of Figure 4c of the main text. It shows that netropsin leads to decreased signal. b) Replotting the data on a log-scale after normalization and fitting exponentials (dashed curves) allows to determine rates. c) Plot of the inversed rates vs. the netropsin concentration and linear fit. The fit shows that the rates decrease with $1 /[$ netropsin]. d) Replot of c) with inversed y-axis. 


\section{Parasite replication kinetics}

To study the parasite replication kinetics, we contaminated the reaction mixtures. For the contaminations we used the parasite that emerged in the untemplated reaction shown in Figure 1d of the main text. Figure S5a shows different dilutions of parasite in a reaction mixture with all nucleotides in the absence of template. For each dilution, the onset reaction time $\tau$ was determined and plotted vs. the decimal logarithm of the dilution factor. A linear fit to this data provided the untemplated replication rate $r=0.05 \mathrm{~min}^{-1}$. The enzyme concentrations were the same as for the experiment of Figure 7 of the main text $(8 \mathrm{U} / \mathrm{ml}$ polymerase, $20 \mathrm{U} / \mathrm{ml}$ nickase and $50 \mathrm{nM}$ exonuclease). The rate of templated replication in Figure 7 for the two non-contaminated samples were $\mathrm{r}=0.04 \mathrm{~min}^{-1}$ and $\mathrm{r}=0.08 \mathrm{~min}^{-1}$, respectively in the presence and in the absence of dATP. Thus, the replication rates of the parasite and of trigger $\mathbf{A}_{1}$ in the presence of $50 \mathrm{nM} \mathbf{T}_{1}$ are comparable. However, $\mathbf{A}_{1}$ is a 12-mer, while the parasite is much longer and thus gives much more signal in the real-time cyclers. In Figure S5b we contaminated an untriggered EXPAR experiment with different dilutions of parasite. In the absence of dATP, high concentrations of parasite have an effect

on templated self-start. In the presence of dATP, templated self-start and untemplated replication cannot be distinguished. 
a)
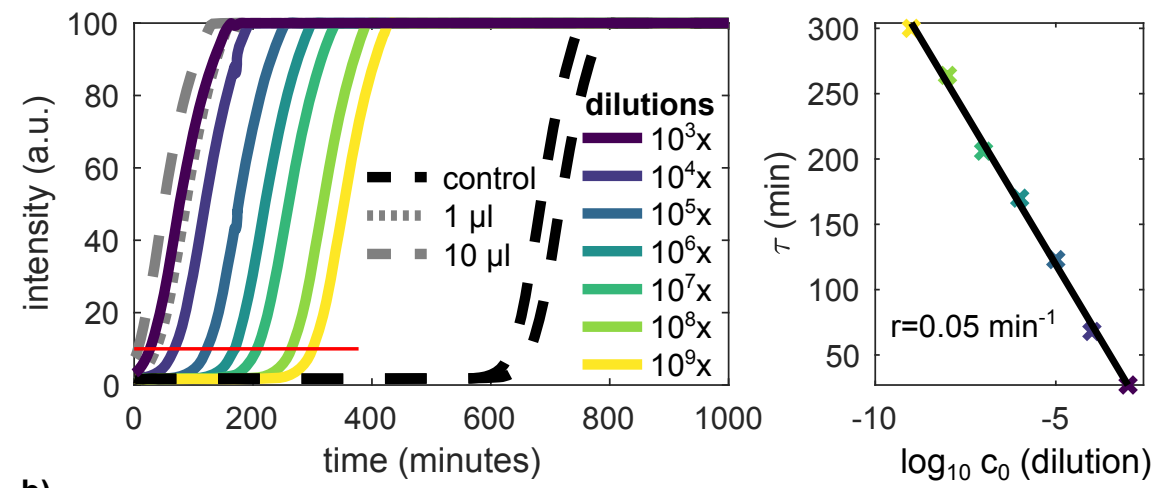

b)
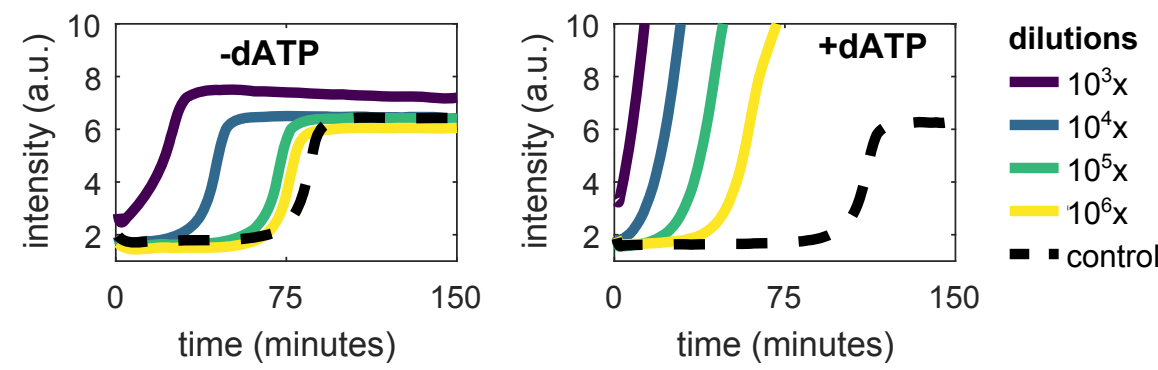

Figure S5: Amplification kinetics of parasite solutions diluted by different factors in the absence and in the presence of template strand. a) A mix containing all nucleotides and enzymes but no template was contaminated with different concentrations of parasite. A $10^{9}$ fold dilution can be easily distinguished from the non-contaminated control (black, dashed lines). For the grey curves, we infected pipette tips with parasite by either filling in $1 \mu \mathrm{l}$ or $10 \mu \mathrm{l}$, emptying them and use them to mix a clean reaction mix. On the right panel we plotted the replication onset time $\tau v s$. the logarithm of the dilution factor to determine the rate of parasite replication. b) In the presence of $50 \mathrm{nM} \mathbf{T}_{1}$ but no trigger $\mathbf{A}_{1}$, only very high concentrations of parasite $\left(\geq 10^{4} \times\right.$ dilution) perturb rEXPAR (-dATP, left) while for standard EXPAR ( + dATP, right) parasite concentrations as low as $\left(\geq 10^{6} \times\right.$ dilution) make it impossible to distinguish EXPAR from parasite replication. 


\section{Bistability}

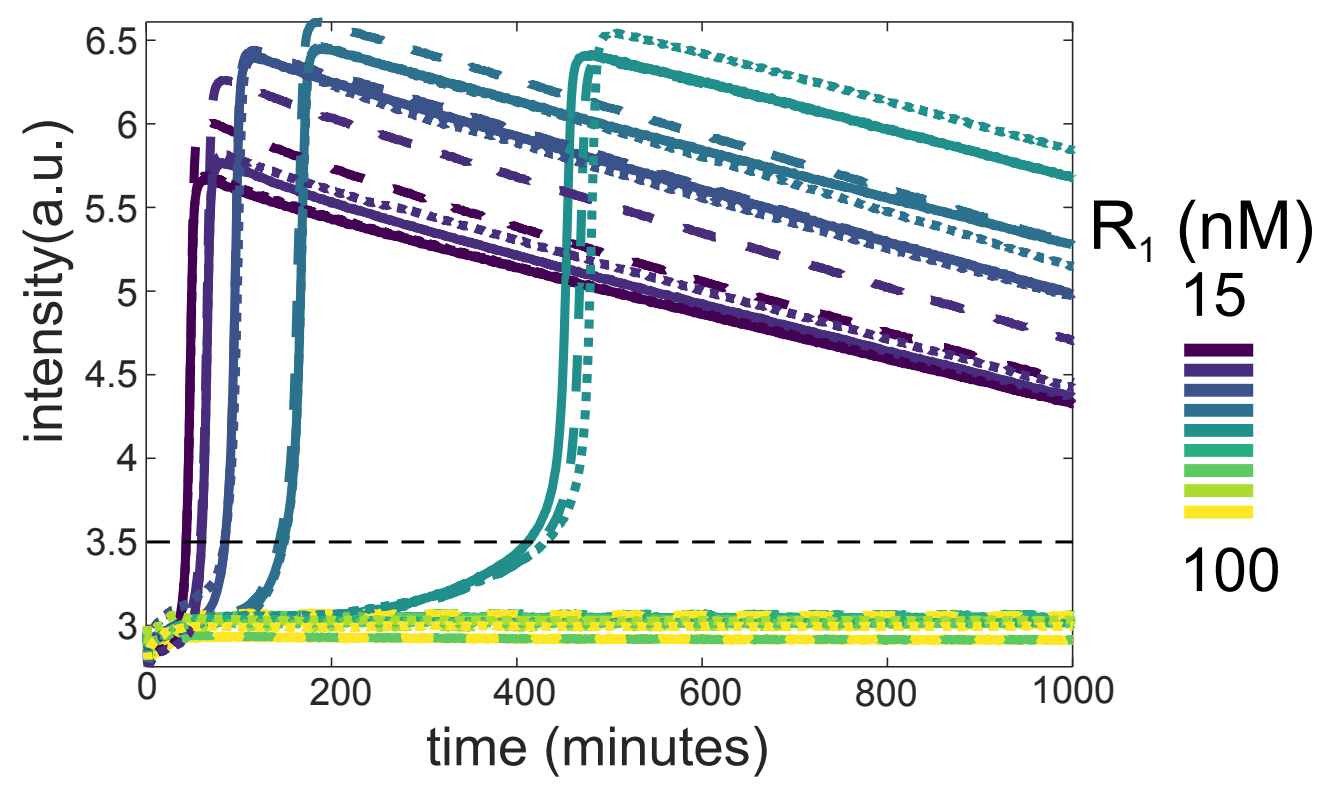

Figure S6: Full data corresponding to Figure 5 in the MT. EvaGreen fluorescence vs. time in the presence of $\mathbf{T}_{1}^{u}$ but in the absence of $\mathbf{A}_{1}$, for increasing concentrations of $\mathbf{R}_{1}$ in $n M$. Experiments performed in the absence of dATP. Colors correspond to different $\mathbf{R}_{1}$ and solid, dashed and dotted lines to three replicates. Conditions: $8 \mathrm{U} / \mathrm{mL}$ pol, $20 \mathrm{u} / \mathrm{mL}$ nick, $100 \mathrm{nM}$ exo and $0.4 \mathrm{mM}$ dNTPs. The signal decreases after amplification because the $\mathbf{T}_{1}^{u}$ template without protection against Nb.BssSI background restriction activity was used. 


\section{Increasing nucleotide concentration slows down tem- plated amplification}

The removal of dATP from the buffer not only prevents parasite formation, but also speeds up the amplification reaction. Figure S7 shows the data for EXPAR experiments for different initial trigger concentrations. In Figure S7a and b on the left an EXPAR experiment is shown with and without dATP, respectively. A range of initial $\mathbf{A}_{1}$ concentration is replicated under otherwise identical conditions, with a trigger-free control (black dashed line). Comparing the graphs shows that the presence of dATP slows down the reaction. To quantify this effect, we plot the time $\tau$ it takes to reach a threshold intensity (red lines) versus the logarithm of the initial trigger concentration c0. From the slopes of the fitted lines we can determine the rates. We get $r=0.90 \mathrm{~min}^{-1}$ in the presence of $\mathrm{dATP}$ and $r=1.51 \mathrm{~min}^{-1}$ in its absence. This is a 1.66-fold increase in replication rate.

In the sequences we used, dATP is not incorporated during replication. Thus, one could argue that this effect is caused by the polymerase being hindered to perform its task by the unusable nucleotides being present in the buffer. However, we also observed a slowing down when the overall amount of nucleotides is reduced (Figure S8). This experiment was done at concentrations of $1 \mathrm{mM}$ per nucleotide with dATP present or removed. In addition, time traces of reactions without dATP and a lower overall nucleotide concentration of $0.2 \mathrm{mM}$ are shown (yellow dashed line). The right graph of the figure shows the initial 200 minutes in more detail. In agreement with the observations made above, the reaction is delayed in the presence of dATP. In addition, comparing the samples without dATP shows that lower dNTPs concentrations speed up the reaction. 


\section{a)}

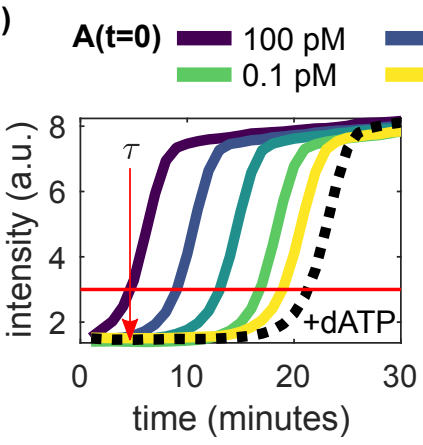

$10 \mathrm{pM}=1 \mathrm{pM}$

$0.01 \mathrm{pM} \quad \boldsymbol{\prime \prime} 0 \mathrm{pM}$

b)
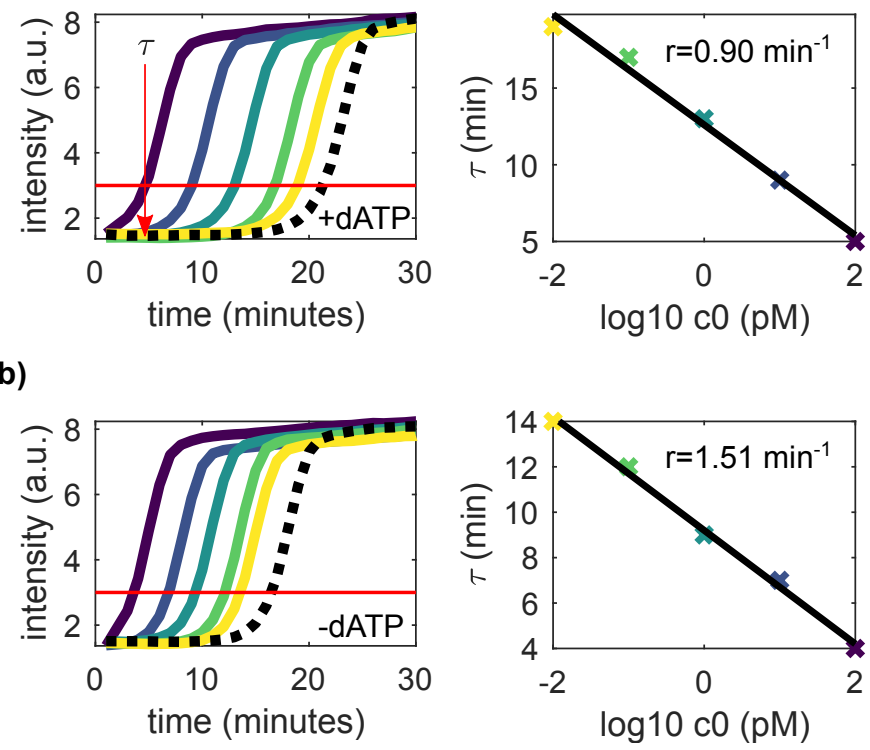

)

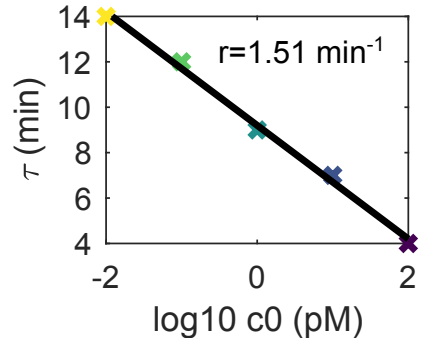

Figure S7: Templated amplification is faster in the absence of dATP. a) The left graph shows a range of initial $\mathbf{A}_{1}$ concentration undergoing an autocatalytic reaction in the presence of dATP. The red horizontal line gives a threshold intensity. The right graph shows the time $\tau$ it takes for each curve to reach the threshold. From this, the replication rate $r$ can be determined by a line fit. b) Shows the same experiment and analysis, but without dATP in the buffer.
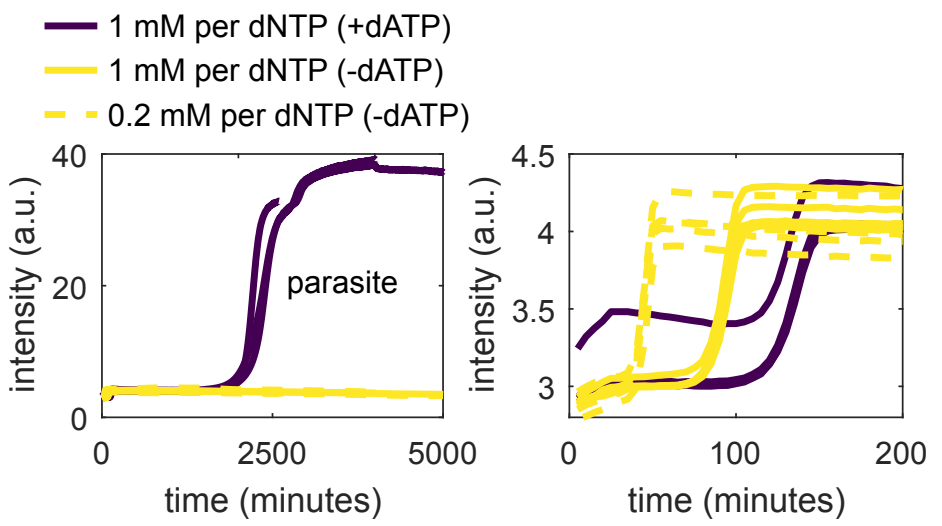

Figure S8: Effects of nucleotide composition and concentration on amplification kinetics. The left graph shows time traces for autocatalysis with $1 \mathrm{mM}$ per nucleotide with and without dATP as well as autocatalysis with $0.2 \mathrm{mM}$ per nucleotide without dATP. The samples were removed from the cycler at different times for PAGE analysis, thus only one time trace per condition stays in the cycler for the full length of the experiment. The right graph shows the first 200 minutes more detailed. The reaction speeds up when dATP is removed and when the overall nucleotide concentration is reduced. The reaction shown is untriggered and contains netrosin. 


\section{Amplification curves for EXPAR and rEXPAR in the presence of parasite contamination}

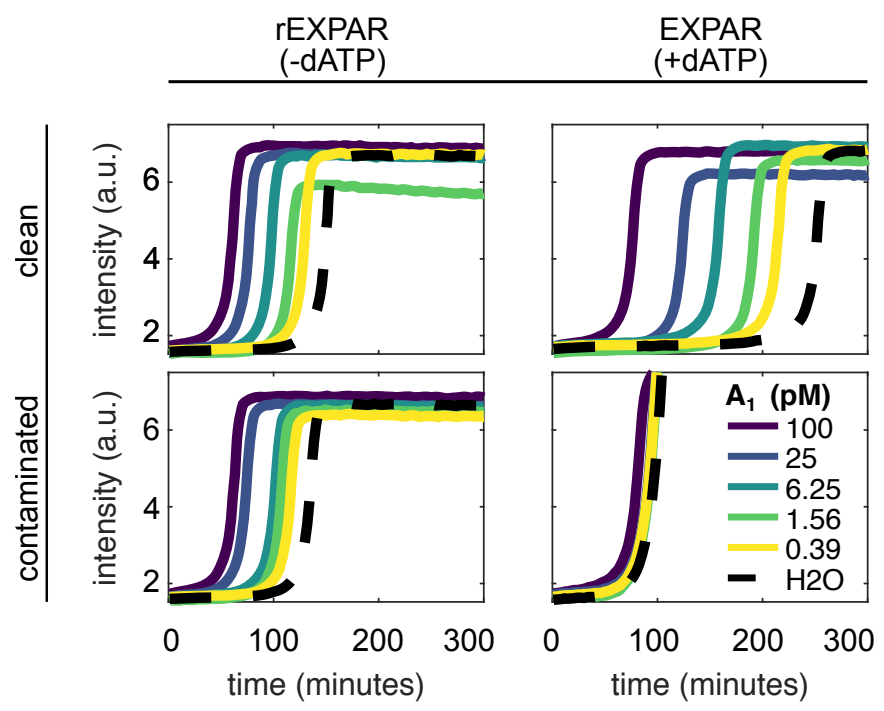

Figure S9: Amplification curves for EXPAR and rEXPAR in the presence of parasite contamination. These graphs show the raw data of Figure 7 of the main text. The threshold used for the analysis was 3 . 